University of Nebraska - Lincoln

DigitalCommons@University of Nebraska - Lincoln

10-1-1986

\title{
Selection rules for vibrational energy loss by resonant electron impact in polyatomic molecules
}

Gordon A. Gallup

University of Nebraska-Lincoln, ggallup1@unl.edu

Follow this and additional works at: https://digitalcommons.unl.edu/physicsgallup

Part of the Physics Commons

Gallup, Gordon A., "Selection rules for vibrational energy loss by resonant electron impact in polyatomic molecules" (1986). Gordon Gallup Publications. 8.

https://digitalcommons.unl.edu/physicsgallup/8

This Article is brought to you for free and open access by the Research Papers in Physics and Astronomy at DigitalCommons@University of Nebraska - Lincoln. It has been accepted for inclusion in Gordon Gallup Publications by an authorized administrator of DigitalCommons@University of Nebraska - Lincoln. 


\title{
Selection rules for vibrational energy loss by resonant electron impact in polyatomic molecules
}

\author{
G. A. Gallup \\ Department of Chemistry, University of Nebraska, Lincoln, Nebraska 68588-0304
}

(Received 18 April 1986)

\begin{abstract}
Approximate expressions for inelastic elements of the $S$ matrix are given in terms of integrals over vibrational functions and $R$-matrix electron states. The magnitude and symmetry of these integrals determine the vibrations that are excited by various resonances, and the present analysis successfully predicts the spectra measured for benzene, ethylene, and formaldehyde. The recently observed $s$-wave component in the rotational excitation of $\mathrm{N}_{2}$ is also explained by the treatment. The theory shows how vibrational excitation can give information about the symmetries of resonant electronic states. This can be particularly useful when angular distribution measurements are unavailable.
\end{abstract}

\section{INTRODUCTION}

Some years ago Wong and Schulz, with a study of benzene, published the first spectra showing vibrational excitation by resonant electron impact in a polyatomic molecule of some complexity. ${ }^{1}$ They observed that only a few of the 30 vibrational modes of benzene are excited after the decay of the temporary negative-ion state (also called a resonance or a compound state) and suggested that a set of highly restrictive, symmetry selection rules operate for this phenomenon. The observed modes could be divided into two types. For one type, the symmetries of the vibrations observed could be attributed to the changed forces on the nuclei from the formation of the temporary negative ion in an undistorted geometry. For the other type, the symmetry of the observed mode was attributed to formation of the negative ion during an "incipient puckering" of the benzene ring, and the scattering showed an isotropic angular distribution characteristic of an $s$ wave. Wong and Schulz go on to say, "This postulate,... tantamount to adding an $s_{\sigma}$ component to the scattered wave, allows us to predict additional allowed modes. ..."

Subsequently, Walker, Stamatovic, and Wong made similar measurements on the somewhat simpler molecule, ethylene. $^{2}$ In their analysis they found the same division of the excited modes into two types, one of which involves an $s$ wave.

Quite recently Benoit and Abouaf examined the vibrational excitation of the still smaller molecule, formaldehyde. $^{3}$ They report the excitation of only one vibrational mode at resonant impact energies and make no mention of the $s$-wave phenomenon. This is in distinct contrast to the results found in all of the hydrocarbon measurements mentioned earlier.

In the present article we give an approximate theoretical description of resonant excitation of vibration and thereby obtain symmetry selection rules for the modes excited. The treatment automatically includes the mechanism involving the above-mentioned $s$ wave. To state the situation more precisely, we will see that, in addition to the principle partial wave of the resonance, a sequence of different vibrational excitations is possible involving $s_{\sigma}, p_{\sigma}, d \sigma, \ldots$ waves, with only the $s_{\sigma}$ expected to be seen at low energies. We shall also suggest that the formaldehyde results are completely consistent with the theory and with the hydrocarbon measurements.

At the outset we may motivate the directions taken in the theoretical treatment by giving a qualitative description of the processes involved. For any experiment in which vibrational excitation accompanies a change in electronic state, the particular vibrations excited are the result of the new forces exerted on the nuclei by the new electronic state produced. This may be treated quantitatively with an application of the Hellmann-Feynman theorem. ${ }^{4}$ In the case of vibrations excited by resonant electron impact, the new forces arise because of the formation of the temporary negative ion. The development will show that the mechanisms giving rise to the two types of vibrations differ only in the particular involvement of the partial waves of the scattered electron. In one case the principal partial wave of the resonance acts alone to excite one particular set of vibrations, and in the other case, the principal partial wave of the resonance interferes or hybridizes with a low- $l$ value (usually $s$ ) nonresonant wave to excite a generally different set of vibrations.

After the development of the theory we give a detailed analysis of the benzene, ethylene, and formaldehyde results referred to above. This will show that the theory accurately predicts the rules operating to select particular vibrational modes in these molecules as well as predicting $s$-wave angular distributions seen in the rotational excitation of $\mathrm{N}_{2}{ }^{5}$ We also predict the expected results for the complex molecules 1,3-butadiene and 1,4-cyclohexadiene, for which the vibrational excitations at resonant impact energies have not yet been determined.

\section{APPROXIMATE $S$ MATRIX}

The "boomerang" model for describing the vibrational structure of shape resonances in molecules has been developed by Herzenberg and his co-workers. ${ }^{6}$ For our 
purposes, however, a time-independent picture seems simpler to develop and we use arguments based upon an $R$-matrix treatment of the electronic state. ${ }^{7}$

We use the adiabatic Born-Oppenheimer model and write the asymptotic wave function in the form

$$
\phi_{a n}=\phi_{0}(r, q) \chi_{a n}(q),
$$

where $\phi_{0}$ is the ground-state electronic wave function, $\chi_{a n}$ is the vibrational (nuclear) wave function, $r$ represents the collection of electronic coordinates, $q$ represents the collection of normal coordinates, and $n$ is the collection of vibrational quantum numbers. Even if the vibrations are anharmonic, we may use the harmonic normal coordinates to describe them.

To describe the $N+1$ electron states we use a method described recently involving basis-set expansion of the $R$ matrix states of the system. ${ }^{8}$ For this a set of one-electron functions, complete inside the sphere, $r=r_{0}$, is used to get

$$
\begin{aligned}
& \Psi_{p}=\underline{A}\left(\phi_{0} w_{p}\right), \\
& \partial\left(r w_{p}\right) /\left.\partial r\right|_{r=r_{0}}=b w_{p},
\end{aligned}
$$

where $\underline{A}$ is the antisymmetrizer and the $R$-matrix functions, $w_{p}$ are expressed as linear combinations in the complete set

$$
w_{p}=\sum_{k, l, m} u_{k l m}^{(0)} a_{k l m ; p} .
$$

Equation (2) represents only an approximate form of the $(N+1)$-electron $R$-matrix function, equivalent to the static exchange approximation. Closed channels giving electron correlation and polarization effects could be included, but these would not change the symmetry of the functions, nor would they change our qualitative conclusions concerning the excitation of vibrations.

Even at low energies, the wave function of the scattered electron may have components with fairly large $l$ values inside the molecule acting as a scattering center. Nevertheless, the low-energy shape resonances for molecules have been successfully interpreted by treating them as if they arise predominantly from a single partial wave. ${ }^{9}$ This is physically reasonable, since, as Fano ${ }^{10}$ has pointed out, outside the region where the potential acts strongly only low- $l$ waves interact appreciably with a localized perturber, even if it is anisotropic. The implication of this for our approximation is that the matrix $a_{k l m ; p}$ associates essentially only one partial wave with each $p$. That is, $\Psi_{p}$ is nearly pure in $l$ and $m$. We now consider the zerothorder set of $w$ functions

$$
w_{p l m}^{(0)}=\sum_{k} u_{k l m}^{(0)} b_{k p}
$$

that would result if only the $l-m$ diagonal blocks of the matrix

$$
\left\langle\underline{A}\left(\phi_{0} u_{k l m}^{(0)}\right)|H(\mathrm{elec})| \underline{A}\left(\phi_{0} u_{k^{\prime} l^{\prime} m^{\prime}}^{(0)}\right)\right\rangle
$$

were diagonalized.

The succeeding argument involves matrix elements that have a large number of subscripts. In order to simplify the notation for the reader we collect the quantum num- bers $p, l, m$, as the single symbol $\alpha$. With this notation the corresponding complete $R$-matrix functions

$$
\Psi_{\alpha}^{(0)}=\underline{A}\left(\phi_{0} w_{\alpha}^{(0)}\right)
$$

give the potentials for the vibrational problem

$$
\begin{aligned}
& V_{\alpha}=\left\langle\Psi_{\alpha}^{(0)}|H(\mathrm{elec})| \Psi_{\alpha}^{(0)}\right\rangle, \\
& {\left[K+\left(V_{\alpha}-V_{\alpha}^{(e)}\right)\right] \chi_{\alpha n}(q)=E_{\alpha n}^{(\mathrm{vib})} \chi_{\alpha n}(q),}
\end{aligned}
$$

where $K$ is the vibrational kinetic energy operator. In (9), $V_{\alpha}^{(e)}$ is the equilibrium $(\partial V / \partial q=0)$ value of the potential. In the succeeding equations we will abbreviate the four quantum numbers $\alpha n=p l m n$ as $\beta$. With this notation, the zeroth-order approximation for the energies of the $R$ matrix poles with vibration is

$$
E_{\beta}^{(t)}=V_{\alpha}^{(e)}+E_{\beta}^{(\mathrm{vib})}
$$

We now proceed to calculate the first-order correction to the wave function.

The nonzero off-diagonal matrix elements of the Hamiltonian are

$$
V_{\beta^{\prime} \beta}=\left\langle\underline{A}\left(\phi_{0} w_{\alpha^{\prime}}^{(0)}\right) \chi_{\beta^{\prime}}|V| \underline{A}\left(\phi_{0} w_{\alpha}^{(0)}\right) \chi_{\beta}\right\rangle,
$$

where $V$ is the potential energy part of the total Hamiltonian. These provide a first-order correction to the total wave function

$$
\begin{aligned}
\Psi_{\beta} & =\Psi_{\beta}^{(0)}+\Psi_{\beta}^{(1)} \\
& =\Psi_{\beta}^{(0)}-\sum_{\beta^{\prime}} \Psi_{\beta^{\prime}}^{(0)} V_{\beta^{\prime} \beta} /\left[E_{\beta^{\prime}}^{(t)}-E_{\beta}^{(t)}\right] \\
& =\sum_{\beta^{\prime}} \Psi_{\beta^{\prime}} U_{\beta^{\prime} \beta} .
\end{aligned}
$$

In (12), $U_{\beta^{\prime} \beta}$ are elements of the matrix, unitary to first order, that gives the transformation from unperturbed to perturbed wave functions. We assume there are no degeneracies among the $E_{\beta}^{(t)}$ so that no first-order corrections to the energy occur. We ignore the second-order corrections.

The standard treatment now gives the $R$ matrix

$$
R_{\gamma^{\prime} \gamma}(E)=\left(\frac{1}{2}\right) \sum_{\beta} W_{\gamma^{\prime} \beta} W_{\gamma \beta} /\left(E_{\beta}^{(t)}-E\right)
$$

where

$$
\begin{gathered}
W_{l^{\prime} m^{\prime} n^{\prime} p l m n}=\sum_{p^{\prime}, n^{\prime \prime}}\left\langle\chi_{a n^{\prime}}\left|\chi_{p^{\prime} l^{\prime} m^{\prime}}^{(0)}\right| \chi_{\rho^{\prime} l^{\prime} m^{\prime} n^{\prime \prime}}\right\rangle \\
\times U_{p^{\prime} l^{\prime} m^{\prime} n^{\prime \prime} p l m n}
\end{gathered}
$$

and $\chi_{p^{\prime} l^{\prime} m^{\prime}}^{(0)}$ is the radial part of $r w_{p^{\prime} l^{\prime} m^{\prime}}^{(0)}$ evaluated at $r=r_{0}$. This is somewhat more complicated if $b \neq 0$.

Measurements of the energy loss have been made with the incoming energy of the electrons set at the resonant value. The flexibility in the $R$ matrix provided by the possibility of adjusting $r_{0}$ [or the value of $b$ in (3)] allows one to arrange a pole of $R$ to be at the resonant energy. Under these circumstances the $S$ matrix takes a particularly simple form

$$
\begin{aligned}
S_{\gamma^{\prime} \gamma}=\delta_{l^{\prime} l} \delta_{m^{\prime} m} \delta_{n^{\prime} n} \epsilon_{\gamma}- & \left(i^{l^{\prime}+l+1} / t_{0}\right) \\
& \times\left(W_{\gamma^{\prime}, 1 l m 0} W_{\gamma, 1 l m 0} / \omega_{\gamma^{\prime}} \omega_{\gamma}\right),
\end{aligned}
$$


where

$$
\begin{aligned}
& \omega_{\gamma}=Y_{\gamma}+i J_{\gamma}, \\
& \epsilon_{\gamma}=\omega_{\gamma}^{*} / \omega_{\gamma}, \\
& t_{0}=\left(\frac{1}{2}\right) W_{p} \omega^{\prime} \omega^{-1} W_{p},
\end{aligned}
$$

and $J_{\gamma}$ and $Y_{\gamma}$ are, respectively, the regular and irregular asymptotic solutions for the channel $\gamma$. In (15) we have labeled the pole of the $R$ matrix at the resonance as $p=1$, but this is clearly not required. Equation (15) is also written for the molecule in its ground vibrational state.

For inelastic scattering events one then has

$$
\left|S_{\gamma^{\prime} \gamma}\right|^{2}=f(E)\left|W_{\gamma^{\prime} \beta}\right|^{2}\left|W_{\gamma \beta}\right|^{2} ; \beta=(1 \operatorname{lm} 0),
$$

where $f(E)$ is a slowly varying function of the asymptotic channel energies. We are particularly interested in situations where electrons in the incoming channel, $\gamma_{i}=\operatorname{lm} 0$, are scattered into the channel, $\gamma_{0}=l^{\prime} m^{\prime} n^{\prime}$. Collecting all of the slowly varying factors into a single, approximately constant proportionality factor we have

$$
\begin{aligned}
& \left|S_{\gamma_{0} \gamma_{i}}\right|^{2} \cong K^{\prime}\left|\left\langle\chi_{a n^{\prime}} \mid \chi_{\beta}\right\rangle\right|^{2} ; l^{\prime} m^{\prime}=\operatorname{lm} ; \beta=1 \operatorname{lm} 0 \\
& \cong K^{\prime} \mid \sum_{p^{\prime \prime}, n^{\prime}} V_{p^{\prime \prime} l^{\prime} m^{\prime} n^{\prime \prime} \beta}\left\langle\chi_{a n^{\prime}} \mid \chi_{p^{\prime \prime} l^{\prime} m^{\prime} n^{\prime \prime}}\right\rangle \\
& \quad \times\left.\left[E_{p^{\prime \prime} l^{\prime} m^{\prime} n^{\prime \prime}}^{(t)}-E_{\beta}^{(0)}\right]^{-1}\right|^{2} ; l^{\prime} m^{\prime} \neq l m,
\end{aligned}
$$

where (17a) refers to the diagonal ( $\mathrm{lm}$ ) blocks, and (17b) for the off-diagonal blocks. The selection rules for vibrational excitation on electron impact at resonant energies in channel $\operatorname{lm} 0$ are embodied in (17). It is seen from the definition of the $V$ integrals that they will be nonzero only if

$$
\underline{A}\left(\phi_{0} w_{p^{\prime} l^{\prime} m^{\prime}}^{(0)}\right) \chi_{p^{\prime} l^{\prime} m^{\prime} n^{\prime}}
$$

and

$$
\underline{A}\left(\phi_{0} w_{p l m}^{(0)}\right) \chi_{p l m n}
$$

belong to the same symmetry species of the molecule point group. It is also seen that $p$ (or $p^{\prime}$ ) merely labels different states of the same symmetry and need not be considered in such arguments. If we are considering exit channels with $l^{\prime} m^{\prime}$ that are not of the correct symmetry to be one of the shape resonances, the Frank-Condon factors $\left\langle\chi_{a n^{\prime}} \mid \chi_{p^{\prime \prime} l^{\prime} m^{\prime} n^{\prime \prime}}\right\rangle$ will be close to $\delta_{n^{\prime} n^{\prime \prime}}$ and approximately independent of the other indices. Thus, the $V$ integrals will govern the selection rules in these cases.

Equation (17) is written in molecule-fixed coordinates and one may remain in this framework to examine the symmetries of vibrational excitation. Should one need to transform to a space-fixed framework, an analysis by Read $^{11}$ is useful.

The two types of terms in (17) both give rise to nonzero cross sections for vibrational excitation. The $\beta$ state in (17a) corresponds to the molecule with one of its antibonding orbitals represented by an $R$-matrix function. An electron occupying this orbital causes forces on the nuclei in directions described by the symmetrized square of the irreducible representation of the state involved. For degenerate states this symmetry analysis is identical with the conventional Jahn-Teller ${ }^{12}$ treatment. For nondegenerate states the symmetrized square gives only the totally symmetric representation and only totally symmetric vibrations will be excited. The Frank-Condon factors in (17a) will reflect the affected vibrational modes. The activity of these modes has already been noted. ${ }^{1}$

The terms from the off-diagonal $\mathrm{lm}$ blocks correspond to interactions between vibration and electronic motion in which different $l$ values mix and the scattered electron appears asymptotically in a different partial wave from that for the resonance. An alternative description is the one in the Introduction: The first-order terms in the wave functions interfere with the zeroth-order terms to give an unsymmetrical charge distribution producing an unsymmetrical set of forces on the nuclei and the excitation corresponding vibration. The forces depend on the amount of mixing and this depends upon the sizes of the corresponding $V$ integrals.

\section{APPLICATIONS}

\section{A. Benzene ${ }^{2} E_{2 u}$ resonance $(1.1 \mathrm{eV})($ Ref. 1)}

For the 1.1-eV resonance of benzene the important input channel is $(\operatorname{lm})=(3, \pm 2)$ of symmetry $e_{2 u}$. The symmetrized square of $e_{2 u}$ is $a_{1 g}+e_{1 g}$. We therefore expect the equilibrium positions in the state $(p \operatorname{lm} 0)=\left(\begin{array}{ll}13 \pm 20 & 3\end{array}\right)$ to be distorted in $A_{1 g}$ and $E_{1 g}$ directions from the asymptotic positions. The Frank-Condon factors in (17a) will be approximately $\delta_{n n^{\prime}}$ except for the $a_{1 g}$ and $e_{1 g}$ species vibrations. This was explained already by Wong and Schulz, ${ }^{1}$ and indeed, $v_{2}$ and $v_{16}$ are seen in the energy-loss spectrum.

As we have seen the excitations of other vibrations can come through the agency of nonzero $V$ integrals in (17b). We first consider outgoing $s$ waves $\left(l^{\prime} m^{\prime} n^{\prime}\right)=\left(00 n^{\prime}\right)$. Vibrations of symmetry $e_{2 u}$ will connect this outgoing channel with the resonant one. $v_{19}$ and $v_{20}$ are the two (doubly degenerate) vibrations of this symmetry, and $v_{20}$, the motion more closely associated with the carbon skeleton distortion, is seen in the energy-loss spectrum. This is to be expected since the $e_{2 u}$ antibonding orbital contains only carbon atomic orbitals (AO's) in the linear combination of AO (LCAO) approximation. A more detailed analysis of $V$ indicates that the progressions $v_{20}+n v_{2}$ and $v_{20}+n v_{16}$ are to be expected. The one based upon $v_{2}$ is actually present; the other is presumably too faint to see.

We next consider exit channels of $l^{\prime}=1$. Since this is not a resonant channel, one expects the $R$-matrix functions to be approximately equal to spherical Bessel functions (that is, they should behave as nearly free electrons). The low- $p$ " terms in (17b) will predominate, and the most important $V$ integrals will be those where $\left(p^{\prime \prime} l l^{\prime} m^{\prime} n^{\prime}\right)$ corresponds to energies close to the electron energy. The $(1 \operatorname{lm} 0)=(13 \pm 20)$ state $\left(e_{2 u}\right)$ is resonant so we expect the $R$-matrix function $w$ to have larger values in the vicinity of the carbon atoms than a free particle $l=3$ function for the same energy would have. The normalization 
constants for Bessel functions satisfying (3) are all approximately equal. Therefore, for $k$ corresponding to $\sim 1 \mathrm{eV}$ and the carbon distance $r_{C} \cong 1.4 \AA$, the value of the $l^{\prime}=1$ function should be $\cong k r_{C} / 3$ smaller than the $l^{\prime}=0$ function in the vicinity of the carbon atoms. The intensity proportional to $V^{2}$ is 0.06 smaller for $p$ waves than $s$ waves, and such vibrations are likely to be difficult to see. A similar analysis for $l^{\prime}=2$ gives an intensity 0.001 lower than the $l^{\prime}=0$ case.

The fact that we expect only $s$-wave outgoing channels for this excitation mechanism implies that a vibrational of the same symmetry as the resonance will be excited. Wong and Schulz have already noted the needed $s$-wave involvement to interpret their results. ${ }^{1}$ The present argument suggests why only $s$ waves are seen at low energies. At higher energies outgoing channels involving $p$ waves may be observed for ideal situations.

Putting together the two excitation mechanisms, for benzene we expect vibrations of symmetry $a_{1 g}, e_{1 g}$, and $e_{2 u}$ to be excited. The one involving greater carbon motion in each of these cases is seen.

\section{B. Benzene ${ }^{2} B_{2 g}$ resonance $(4.8 \mathrm{eV})$}

Following the principles of Sec. III A, one expects $a_{1 g}$ and $b_{2 g}$ vibrations to be excited. Since an $l^{\prime}=4$ partial wave of low energy should peak so far from the center of the molecule, $\mathrm{C}-\mathrm{H}$ rather than $\mathrm{C}-\mathrm{C}$ deformations appear more strongly excited, and $v_{1}$ and $v_{7}$ are seen in the spectrum. Since there is no clear progression involving $a_{1 g}$, the shift in carbon positions for this resonance must be minimal.

The vibrational energy-loss spectra leading to $l^{\prime}=1$ exit channels are expected to be $\sim 0.25$ less intense than those leading to $l^{\prime}=0$ channels for the ${ }^{2} B_{2 g}$ resonance. The symmetries of the vibrations interacting with outgoing $p$ waves are $b_{1 g}$, which is not represented, and $e_{2 u}$, which is represented by $v_{19}$ and $v_{20}$, as we have seen. $v_{19}$ is within $50 \mathrm{meV}$ of $v_{7}$ and, at $\frac{1}{4}$ the intensity, may be obscured. The $d$-wave intensity is down by a factor of 0.01 and need not be considered.

\section{Ethylene ${ }^{2} B_{2 g}$ resonance $(1.8 \mathrm{eV})$ (Ref. 2)}

For ethylene, ${ }^{13}$ the present analysis predicts the occurrence of $a_{1 \mathrm{~g}}$ and $b_{2 g}$ vibrations. All three of the $a_{1 \mathrm{~g}}$ frequencies, $v_{1}, v_{2}$, and $v_{3}$ are observed. $v_{2}$, the mode with the greatest $\mathrm{C}-\mathrm{C}$ stretch component, is the strongest. Walker et al. attribute another peak to $v_{7}$, the ir active out-of-plane $\mathrm{CH}_{2}$ wagging mode. The present analysis suggests this should be $v_{8}$, the Raman-active mode of the same type. The two frequencies are $6 \mathrm{~cm}^{-1}$ apart, a difference not detectable in these experiments. In their $\mathrm{C}_{2} \mathrm{D}_{4}$ measurements the difference between $v_{7}$ and $v_{8}$ is 60 $\mathrm{cm}^{-1}$, still very small at $7 \mathrm{meV}$. The excitations leading to $l^{\prime}=1$ frequencies are predicted to be $\sim 0.02$ less intense.

The angular distribution of the peak we attribute to $v_{8}$ shows a nearly isotropic distribution of an outgoing $s$ wave character. The other peaks in the energy loss at 1.8
eV show a strong angular dependence indicative of the " $d$-wave" resonance.

\section{Ethylene, $7.5 \mathrm{eV}$ "resonance" (Ref. 2)}

Walker et al. also finds a broad feature at $7.5 \mathrm{eV}$ in $v_{1}$ energy loss. They attribute this to the unoccupied $4 a_{1 g} \sigma$ orbital, which is predominantly antibonding at the $\mathrm{C}-\mathrm{H}$ bonds. Such a mechanism is not likely to be well described by just a few terms of (17).

There is also a peak in the energy-loss spectrum at this impact energy that Walker et al. attribute to $v_{7}$ with a possibly twisted or distorted geometry invoked. In light of the present treatment, we suggest this is just $v_{8}\left(b_{2 g}\right)$ again, excited this time by a reverse $s \rightarrow d$-wave process compared to that occurring at $1.8 \mathrm{eV}$. Although the outgoing wave for this mechanism is nominally $d$, the coupling of an $s$ incoming channel with a random average over molecule orientations will produce an isotropic distribution as shown in Fig. 6 of Ref. 2.

\section{E. Formaldehyde ${ }^{2} B_{1}$ resonance $(0.65 \mathrm{eV})$ (Ref. 3)}

Benoit and Abouaf have recently determined the electron energy-loss spectrum of formaldehyde at an impact energy of $0.8 \mathrm{eV}$ and an observation angle of $90^{\circ}$. They report the expected presence of the $\mathrm{C}=\mathrm{O}$ stretch $v_{2}$, which is excited because the resonance has $b_{2}$ symmetry and is associated with the $\mathrm{C}-\mathrm{O}$ antibonding $\pi$ orbital. ${ }^{14}$ The theory given here also predicts that $v_{6}, \mathrm{C} \mathrm{CH}_{2}$ wag mode with symmetry $b_{2}$, should be seen. Benoit and Abouaf do not report an energy loss in this mode. In neutral $\mathrm{CH}_{2} \mathrm{O} v_{2}$ has an energy of $216 \mathrm{meV}$ and $v_{6}$ an energy of $145 \mathrm{meV}$. A careful examination of Fig. 1 of Ref. 3 shows two shoulders of the $v_{2}$ peak that are in the correct place for $v_{6}$ and $2 v_{6} .3 v_{6}$ is likely to be completely obscured by $2 v_{2}$. It is suggested here that these are the energy-loss peaks associated with $v_{6}$ predicted by the present theory.

Benoit and Abouaf attribute one of these shoulders to $\mathrm{CO}$ as an impurity. We feel that this can be ruled out because of the low probability of vibrational excitation of $\mathrm{CO}$ at an impact energy of $0.8 \mathrm{eV}$ and an observation angle of $90^{\circ} .{ }^{15}$ Similar measurements of HDCO and $\mathrm{D}_{2} \mathrm{CO}$ could resolve this question.

\section{F. Some other hydrocarbons}

Resonances have been observed in the total scattering cross section of other hydrocarbons, ${ }^{9}$ but not the vibrational energy loss at resonant impact energies. Two of these for which the vibrational frequencies of the neutral molecules have been assigned are 1,3-butadiene ${ }^{16}$ and 1,4cyclohexadiene. ${ }^{17}$ The resonances for 1,3-butadiene are given by Burrow and Jordan ${ }^{18}$ and those for 1,4cyclohexadience by Jordan, Michejda, and Burrow. ${ }^{19}$ The present analysis may be applied to each of these and gives the following predictions.

The lower resonance for 1,3-butadiene in transoid $\left(C_{2 h}\right)$ conformation is of symmetry ${ }^{2} A_{\boldsymbol{u}}$ with a leading $l$ value of 1 . We expect an $a_{g}$ vibration (probably $v_{4} \mathrm{C}=\mathrm{C}$ stretch) energy loss with $p$-wave angular distribution and 
an $a_{u}$ vibration (probably $v_{10}$ or $v_{11} \mathrm{CH}_{2}$ wagging) with an $s$-wave angular distribution. The upper resonance is ${ }^{2} B_{g}$ with a leading $l$ value of 2 . We expect an $a_{g}$ vibration (probably $v_{4}$ again) with a $d$-wave angular distribution and a $b_{g}$ vibration (probably $v_{15} \mathrm{CH}_{2}$ wagging) with an $s$ wave angular distribution.

For 1,4-cyclohexadiene $\left(D_{2 h}\right)$ the two close resonances are expected to be of ${ }^{2} B_{3 g}$ and ${ }^{2} A_{u}$ symmetries, with leading $l$ values of 2 and 3 , respectively. The first will give vibrational excitations of symmetries $a_{g}$ (probably $v_{8 a} \mathrm{C}=$ $\mathrm{C}$ stretch) and $b_{3 g}$ (probably $v_{5}$ out of plane $\mathrm{C}=\mathrm{C}$ bend) symmetries. The second will also give $a_{g}\left(v_{8 a}\right)$ again and $a_{u}$ (probably $v_{17 a}$ out of plane $\mathrm{C}=\mathrm{C}$ bend) vibrations. In each case the $a_{g}$ vibration will show the angular distribution of the electronic resonance and the other will show an $s$-wave distribution.

\section{G. Rotationally inelastic collisions}

Equation (17) applies with only a slight modification to rotational energy-loss processes, also. At normal temperatures rotational superelastic events are also possible. The analysis is much the same as for vibrations, since rotational motion, may also be classified with respect to the symmetry species of the molecule. The results may be modified by nuclear statistics. ${ }^{20}$

In benzene the symmetries of the resonances $e_{2 u}$ and $b_{2 g}$ are not represented by rotations so that no involvement is expected here. The $b_{2 g}$ resonance of ethylene is the species of a rotation, and, therefore, (17b)-type interactions should produce rotational energy loss or gain with an $s$-wave distribution.

With respect to the symmetry of rotations, $\mathrm{N}_{2}$ and ethylene are similar. A recent study of rotational excitation in $N_{2}$ by Jung et al. ${ }^{5}$ shows an $s$-wave component in the angular distribution. We suggest that this is due to terms of the type $(17 \mathrm{~b})$ in the cross section. The $b_{2}$ resonance in formaldehyde should also exhibit $s$-wave components in the rotational excitation spectrum.

In 1,3-butadiene the $B_{g}$ resonance and in 1,4cyclohexadiene the $B_{3 g}$ resonance should show rotational $s$-wave distributions.

\section{SUMMARY}

The present article has given a first-order perturbation treatment of approximate $R$-matrix functions. The theory has been used to study vibrational excitation at resonant electron energies. The excitation of various vibrational modes arises from the forces on the nuclei resulting from the formation of the temporary negative ion. These forces are best understood in terms of the Hellman-Feynman theorem. At low energies two somewhat different mechanisms for excitation are expected. In the first, the principal partial wave in which the resonance occurs acts alone to produce forces on the nuclei and the excitation of a corresponding set of vibrational modes. In the second, the principal partial wave in which the resonance occurs interferes or hybridizes with the nonresonant $s$ wave to produce a, in general, different set of nuclear forces and corresponding mode excitations.

In the second case the mechanism produces outgoing $s$ waves and has the possibility of giving information concerning the symmetries of resonances in cases where angular distributions are unavailable.

\section{ACKNOWLEDGMENT}

The author wishes to express his sincere appreciation to P. D. Burrow and J. H. Macek for many useful discussions concerning this work.
${ }^{1}$ S. F. Wong and G. J. Schulz, Phys. Rev. Lett. 35, 1429 (1975).

${ }^{2}$ I. C. Walker, A. Stamatovic, and S. F. Wong, J. Chem. Phys. 69, 5532 (1978).

${ }^{3}$ C. Benoit and R. Abouaf, Chem. Phys. Lett. 123, 134 (1986).

${ }^{4}$ See, for example, B. M. Deb, Rev. Mod. Phys. 45, 22 (1973).

${ }^{5}$ K. Jung, T. Antoni, R. Mueller, K.-H. Kochem, and H. Ehrhardt, J. Phys. B 15, 3535 (1982).

${ }^{6}$ D. T. Birtwistle and A. Herzenberg, J. Phys. B 4, 53 (1971); L. Dube and A. Herzenberg, Phys. Rev. A 11, 1314 (1975).

${ }^{7}$ E. P. Wigner and L. Eisenbud, Phys. Rev. 72, 29 (1947); see also, T. Y. Wu and T. Ohmura, Quantum Theory of Scattering (Prentice-Hall, Englewood Cliffs, 1962), Chap. 6.

${ }^{8}$ B. I. Schneider, Phys. Rev. A 14, 1923 (1976); B. I. Schneider, M. Le Dourneuf, and P. G. Burke, J. Phys. B 12, L365 (1979).

${ }^{9}$ See, for example, K. D. Jordan and P. D. Burrow, Acc. Chem. Res. 11, 34 (1978).

${ }^{10}$ U. Fano, Phys. Rev. Lett. 31, 234 (1973).

${ }^{11}$ F. H. Read, J. Phys. B 1, 893 (1968).
${ }^{12}$ R. Englman, Jahn-Teller Effect in Molecules and Crystals (Wiley, New York, 1972).

${ }^{13} \mathrm{We}$ use the numbering and symmetries of G. Herzberg, Infrared and Raman Spectra (Van Nostrand, New York, 1945).

${ }^{14}$ P. D. Burrow and J. A. Michejda, Chem. Phys. Lett. 42, 223 (1976).

${ }^{15}$ H. Ehrhardt, L. Langhans, F. Linder, and H. S. Taylor, Phys. Rev. 173, 222 (1968).

${ }^{16}$ T. Shimanouchi, Tables of Molecular Vibration Frequencies, Natl. Bur. Stand. Ref. Data Ser., Natl. Bur. Stand. (U.S.) Circ. No. 39 (U.S. GPO Washington, D.C., 1972).

${ }^{17}$ H. D. Stidham, Spectrochim. Acta 21, 23 (1965).

18P. D. Burrow and K. D. Jordan, Chem. Phys. Lett. 36, 594 (1975).

${ }^{19}$ K. D. Jordan, J. A. Michejda, and P. D. Burrow, Chem. Phys. Lett. 42, 227 (1976).

${ }^{20}$ G. A. Natanson, J. Phys. B 18, 4481 (1985). 